\title{
Viscosity Approximation Methods for Two Accretive Operators in Banach Spaces
}

\author{
Jun-Min Chen and Tie-Gang Fan \\ College of Mathematics and Computer, Hebei University, Baoding 071002, China \\ Correspondence should be addressed to Jun-Min Chen; chenjm130@gmail.com
}

Received 19 July 2013; Revised 24 October 2013; Accepted 25 October 2013

Academic Editor: Somyot Plubtieng

Copyright (C) 2013 J.-M. Chen and T.-G. Fan. This is an open access article distributed under the Creative Commons Attribution License, which permits unrestricted use, distribution, and reproduction in any medium, provided the original work is properly cited.

We introduced a viscosity iterative scheme for approximating the common zero of two accretive operators in a strictly convex Banach space which has a uniformly Gâteaux differentiable norm. Some strong convergence theorems are proved, which improve and extend the results of Ceng et al. (2009) and some others.

\section{Introduction and Preliminaries}

Let $E$ be a real Banach space, $C$ a nonempty closed convex subset of $E$, and $T: C \rightarrow C$ a mapping. Recall that $T$ is nonexpansive if $\|T x-T y\| \leq\|x-y\|$, for all $x, y \in C$. A point $x \in C$ is a fixed point of $T$ provided that $T x=x$. Denote by $F(T)$ the set of fixed points of $T$; that is, $F(T)=$ $\{x \in C, T x=x\}$. Throughout this paper, we assume that $T$ is a nonexpansive mapping such that $F(T) \neq \varnothing$. Recall that a self-mapping $f: C \rightarrow C$ is a contraction on $C$ if there exists a constant $\alpha \in(0,1)$ such that $\|f(x)-f(y)\| \leq$ $\alpha\|x-y\|$, for all $x, y \in C$. Let $\Sigma_{C}=\{f: C \rightarrow C$ । $f$ is a contraction with constant $\alpha$ \}. The normalized duality mapping $J$ from $E$ into $2^{E^{*}}$ is given by $J(x)=\left\{f \in E^{*}\right.$ : $\left.\langle x, f\rangle=\|x\|^{2}=\|f\|^{2}\right\}, x \in E$, where $E^{*}$ denotes the dual space of $E$ and $\langle\cdot, \cdot\rangle$ denotes the generalized duality pairing.

A Banach space $E$ is said to be strictly convex if $\|(x+$ $y) / 2 \|<1$, for all $x \neq y \in E$ with $\|x\|=\|y\|=1$. It is said to be uniformly convex if $\lim _{n \rightarrow \infty}\left\|x_{n}-y_{n}\right\|=0$, for any two sequences $\left\{x_{n}\right\},\left\{y_{n}\right\}$ in $E$ such that $\|x\|=\|y\|=1$ and $\lim _{n \rightarrow \infty}\left(\left\|x_{n}+y_{n}\right\| / 2\right)=1$.

The norm of $E$ is said to be Gâteaux differentiable if

$$
\lim _{t \rightarrow 0} \frac{\|x+t y\|-\|x\|}{t}
$$

exists for each $x, y$ in its unit sphere $U=\{x \in E,\|x\|=1\}$. Such an $E$ is called a smooth Banach space. The norm is said to be uniformly Gâteaux differentiable if, for each $y \in U$, the limit is attained uniformly for $x \in U$. It is well known that $E$ is smooth if and only if the duality mapping $J$ is single valued and that, if $E$ has a uniformly Gâteaux differentiable norm, $J$ is uniformly norm to weak ${ }^{*}$ continuous on each bounded subset of $E$ (cf. [1]).

Let $D$ be a subset of $C$. Then $Q: C \rightarrow D$ is called a retraction from $C$ onto $D$ if $Q(x)=x$ for all $x \in D$. A retraction $Q: C \rightarrow D$ is said to be sunny if $Q(Q x+t(x-$ $Q x))=Q x$ for all $x \in C$ and $t \geq 0$ whenever $Q x+t(x-Q x) \epsilon$ $C$. A subset $D$ of $C$ is said to be a sunny nonexpansive retract of $C$ if there exists a sunny nonexpansive retraction of $C$ onto $D$. In a smooth Banach space $E$, it is known that $Q: C \rightarrow D$ is a sunny nonexpansive retraction if and only if the following condition holds (cf. [2, page 48]):

$$
\langle x-Q x, J(z-Q x)\rangle \leq 0, \quad x \in C, z \in D .
$$

Recall that an operator $A$ with $D(A)$ and $R(A)$ in $E$ is said to be accretive if, for each $x_{i} \in D(A)$ and $y_{i} \in A x_{i}, i=1,2$, there exists a $j \in J\left(x_{2}-x_{1}\right)$ such that $\left\langle y_{2}-y_{1}, j\right\rangle \geq 0$. An accretive operator $A$ is $m$-accretive if $R(I+\lambda A)=E$, for all $\lambda>0$. Denote by $A^{-1} 0$ the set of zeros of $A$; that is, $A^{-1} 0=$ $\{x \in D(A), A x=0\}$.

Denote by $J_{r}(r>0)$ the resolvent of $A$; that is, $J_{r}=(I+$ $r A)^{-1}$. It is well known that $F\left(J_{r}\right)=A^{-1} 0$, for all $r>0$. And if $D(A)$ is convex, then $J_{r}$ is a nonexpansive mapping from $E$ to 
$D(A)$. If $E$ is a Hilbert space, then $A$ is a maximal monotone operator if and only if $A$ is an $m$-accretive operator.

Recently, the approximation of zeros of accretive operators has been studied extensively (see, e.g., [3-9]). Specially, Ceng et al. [10] studied the following composite iterative scheme in uniformly smooth Banach spaces:

$$
\begin{gathered}
y_{n}=\alpha_{n} u+\left(1-\alpha_{n}\right) J_{r_{n}} x_{n}, \\
x_{n+1}=\beta_{n} y_{n}+\left(1-\beta_{n}\right) J_{r_{n}} y_{n},
\end{gathered}
$$

where $u \in \overline{D(A)}$ is an arbitrary (but fixed) element. They proved that $\left\{x_{n}\right\}$ generated by (3) converges strongly to a zero of $m$-accretive operator $A$ under certain appropriate conditions.

Very recently, Chen et al. [11] considered the following viscosity iterative scheme in a reflexive Banach space having a weakly sequentially continuous duality mapping:

$$
\begin{gathered}
y_{n}=\alpha_{n} x_{n}+\left(1-\alpha_{n}\right) J_{r_{n}} x_{n}, \\
x_{n+1}=\beta_{n} f\left(x_{n}\right)+\left(1-\beta_{n}\right) y_{n},
\end{gathered}
$$

where $\left\{\alpha_{n}\right\},\left\{\beta_{n}\right\} \subset(0,1)$. Under some conditions, they showed that $\left\{x_{n}\right\}$ generated by (4) converges strongly to a zero of $m$-accretive operator $A$.

In this paper, motivated by [10-14], we will consider the following so-called composite viscosity iterative scheme for finding a common zero of two $m$-accretive operators:

$$
\begin{aligned}
& y_{n}=\alpha_{n} f\left(x_{n}\right)+\left(1-\alpha_{n}\right) S_{r_{n}} x_{n}, \\
& x_{n+1}=\beta_{n} f\left(y_{n}\right)+\left(1-\beta_{n}\right) y_{n},
\end{aligned}
$$

where $A$ and $B$ are $m$-accretive operators, $S_{r_{n}}=(1-\lambda) J_{r}^{A}+\lambda J_{r}^{B}$ such that $F\left(S_{r}\right)=A^{-1} 0 \cap B^{-1} 0 \neq \varnothing, C=\overline{D(A)}=\overline{D(B)}$, $f \in \Sigma_{C}$, and $\left\{\alpha_{n}\right\},\left\{\beta_{n}\right\} \subset(0,1)$. Under some conditions, we will prove that $\left\{x_{n}\right\}$ generated by (5) converges strongly to a common zero of $A$ and $B$ in a strictly convex and reflexive Banach space having a uniformly Gâteaux differentiable norm, which improve the corresponding results in [10-13].

Lemma 1 (see [10]). In a Banach space E, the following inequality holds:

$$
\|x+y\|^{2} \leq\|x\|^{2}+2\langle y, j(x+y)\rangle, \quad \forall x, y \in E,
$$

where $j(x+y) \in J(x+y)$.

Lemma 2 (see $[10,13])$. Let $\left\{\alpha_{n}\right\}$ be a sequence of nonnegative real numbers satisfying the condition

$$
\alpha_{n+1} \leq\left(1-\gamma_{n}\right) \alpha_{n}+\sigma_{n} \gamma_{n}, \quad \forall n \geq 0,
$$

where $\left\{\gamma_{n}\right\} \subset(0,1)$ and $\left\{\sigma_{n}\right\}$ such that

(i) $\lim _{n \rightarrow \infty} \gamma_{n}=0$ and $\sum_{n=1}^{\infty} \gamma_{n}=\infty$;

(ii) either $\lim \sup _{n \rightarrow \infty} \sigma_{n} \leq 0$ or $\sum_{n=1}^{\infty}\left|\gamma_{n} \sigma_{n}\right|<\infty$.

Then $\lim _{n \rightarrow \infty} \alpha_{n}=0$.
Lemma 3 (the resolvent identity [10]). For $\lambda>0, \mu>0$ and $x \in E$,

$$
J_{\lambda} x=J_{\mu}\left(\frac{\mu}{\lambda} x+\left(1-\frac{\mu}{\lambda}\right) J_{\lambda} x\right)
$$

Lemma 4 (see [3, Theorem 4.1, page 287]). Let $E$ be a uniformly smooth Banach space, $C$ be a closed convex subset of $E, T: C \rightarrow C$ a nonexpansive mapping with $F(T) \neq \varnothing$, and $f \in \Sigma_{C}$. Then $\left\{z_{t}\right\}$ defined by the following

$$
z_{t}=t f\left(z_{t}\right)+(1-t) T z_{t}, \quad z_{t} \in C
$$

converges strongly to a point in $\operatorname{Fix}(T)$. If, moreover, one defines $Q: \Sigma_{C} \rightarrow F(T)$ by

$$
Q(f):=\lim _{t \rightarrow 0} z_{t}, \quad f \in \Sigma_{C},
$$

then $Q(f)$ solves the variational inequality

$$
\begin{array}{r}
\langle(I-f) Q(f), J(Q(f)-p)\rangle \leq 0, \\
f \in \Sigma_{C}, \quad p \in F(T) .
\end{array}
$$

Recall that a mapping $g: C \rightarrow C$ is said to be weakly contractive $[15,16]$ if

$$
\|g(x)-g(y)\| \leq\|x-y\|-\psi(\|x-y\|), \quad \forall x, y \in C,
$$

where $\psi:[0,+\infty) \rightarrow[0,+\infty)$ is a continuous and strictly increasing function such that $\psi$ is positive on $(0,+\infty)$ and $\psi(0)=0$. As a special case, if $\psi(t)=(1-k) t$ for $t \in[0,+\infty)$, where $k \in(0,1)$, then the weakly contractive mapping $g$ is a contraction with constant $k$. Rhodes [17] obtained the following result for weakly contractive mapping (see also [16]).

Lemma 5 (see [17, Theorem 2]). Let $(X, d)$ be a complete metric space and $g$ a weakly contractive mapping on $X$. Then $g$ has a unique fixed point $p$ in $X$.

Lemma 6. Let $\left\{s_{n}\right\}$ and $\left\{\gamma_{n}\right\}$ be two sequences of nonnegative real numbers and $\left\{\lambda_{n}\right\}$ a sequence of positive numbers satisfying the conditions

(i) $\sum_{n=1}^{\infty} \lambda_{n}=+\infty$,

(ii) $\lim _{n \rightarrow \infty}\left(\gamma_{n} / \lambda_{n}\right)=0$.

Let the recursive inequality

$$
s_{n+1} \leq s_{n}-\lambda_{n} \psi\left(s_{n}\right)+\lambda_{n} \gamma_{n}, \quad n=0,1,2, \ldots,
$$

be given where $\psi(t)$ is a continuous and strict increasing function on $[0,+\infty)$ with $\psi(0)=0$. Then $\lim _{n \rightarrow \infty} s_{n}=0$.

\section{Main Results}

Throughout this section, we assume the following.

(i) $E$ is a strictly convex Banach space which has a uniformly Gâteaux differentiable norm, and $C$ is a nonempty closed convex subset of $E$. 
(ii) Take $S_{r}=(1-\lambda) J_{r}^{A}+\lambda J_{r}^{B}, 0<\lambda<1$. Obviously $S_{r}$ is nonexpansive mapping and $F\left(S_{r}\right)=A^{-1} 0 \cap B^{-1} 0$, if $E$ is a strictly convex Banach space. Indeed, it is easy to see that $F\left(S_{r}\right) \supset A^{-1} 0 \cap B^{-1} 0$. Let $q \in F\left(S_{r}\right), p \in A^{-1} 0 \cap B^{-1} 0$; then

$$
\begin{aligned}
\|q-p\| & \leq(1-\lambda)\left\|J_{r}^{A} q-p\right\|+\lambda\left\|J_{r}^{B} q-p\right\| \\
& \leq(1-\lambda)\left\|J_{r}^{A} q-p\right\|+\lambda\|q-p\| \leq\|q-p\| .
\end{aligned}
$$

From the above formula, we obtain $(1-\lambda)\left\|J_{r}^{A} q-p\right\|+\lambda\|q-p\|=$ $\|q-p\|$, so $\left\|J_{r}^{A} q-p\right\|=\|q-p\|$. Similarly, $\left\|J_{r}^{B} q-p\right\|=\|q-p\|$. But

$$
\|q-p\|=\left\|(1-\lambda)\left(J_{r}^{A} q-p\right)+\lambda\left(J_{r}^{B} q-p\right)\right\| .
$$

Then the strict convexity of $E$ implies that $q-p=J_{r}^{A} q-p=$ $J_{r}^{B} q-p$, that is, $q=J_{r}^{A} q=J_{r}^{B} q$, or $q \in A^{-1} 0 \cap B^{-1} 0$.

Theorem 7. Let $E$ be a strictly convex Banach space which has a uniformly Gâteaux differentiable norm, $A, B$ two $m$-accretive maps in $E$ such that $C=\overline{D(A)}=\overline{D(B)}$ is convex and $A^{-1} 0 \cap$ $B^{-1} 0 \neq \varnothing$, and $f: C \rightarrow C$ a fixed contraction mapping with contract constant $\alpha$. Suppose that $\alpha_{n} \subset(0,1), \beta_{n} \subset(0,1)$, and $r_{n}>0$ satisfy the following conditions:

(i) $\sum_{n=1}^{\infty} \alpha_{n}=\infty, \alpha_{n} \rightarrow 0$, as $n \rightarrow \infty$;

(ii) $\beta_{n} \rightarrow 0$, and $r_{n} \rightarrow r>\varepsilon>0$ as $n \rightarrow \infty$;

(iii) $\begin{aligned} \sum_{n=1}^{\infty}\left|\alpha_{n+1}-\alpha_{n}\right|<\infty, \sum_{n=1}^{\infty}\left|\beta_{n+1}-\beta_{n}\right|<\infty \text {, and } \\ \\ \sum_{n=1}^{\infty}|| r_{n+1}-r_{n} \mid<\infty .\end{aligned}$

Let $\left\{x_{n}\right\}$ be the composite viscosity process defined by

$$
\begin{aligned}
& y_{n}=\alpha_{n} f\left(x_{n}\right)+\left(1-\alpha_{n}\right) S_{r_{n}} x_{n}, \\
& x_{n+1}=\beta_{n} f\left(y_{n}\right)+\left(1-\beta_{n}\right) y_{n} .
\end{aligned}
$$

Then $\left\{x_{n}\right\}$ converges strongly to $p \in A^{-1} 0 \cap B^{-1} 0$, where $p$ is the unique solution of the following variational inequality:

$$
\langle(I-f) p, J(p-q)\rangle \leq 0, \quad f \in \Sigma_{C}, q \in A^{-1} 0 \cap B^{-1} 0 .
$$

Proof. First, by using Lemma 4, we know that there exists the unique solution $p$ of a variational inequality

$$
\langle(I-f) p, J(p-q)\rangle \leq 0, \quad f \in \Sigma_{C}, q \in A^{-1} 0 \cap B^{-1} 0,
$$

where $p=\lim _{t \rightarrow 0} z_{t}$ and $z_{t}$ is defined by $z_{t}=t f\left(z_{t}\right)+(1-$ $t) S_{r}\left(z_{t}\right)$ for each $r>0$ and $0<t<1$.

Next, we will divide our discussion into the following steps.

Step 1. We will show that $\left\{x_{n}\right\}$ is bounded.

In fact, take $p \in A^{-1} 0 \cap B^{-1} 0$. Then

$$
\begin{aligned}
\left\|y_{n}-p\right\| & =\left\|\alpha_{n} f\left(x_{n}\right)+\left(1-\alpha_{n}\right) S_{r_{n}} x_{n}-p\right\| \\
& \leq\left(1-\alpha_{n}(1-\alpha)\right)\left\|x_{n}-p\right\|+\alpha_{n}\|f(p)-p\| .
\end{aligned}
$$

Therefore,

$$
\begin{aligned}
\left\|x_{n+1}-p\right\|= & \left\|\beta_{n} f\left(y_{n}\right)+\left(1-\beta_{n}\right) y_{n}-p\right\| \\
\leq & \left(1-\beta_{n}(1-\alpha)\right)\left\|y_{n}-p\right\|+\beta_{n}\|f(p)-p\| \\
\leq & {\left[1-(1-\alpha)\left(\beta_{n}+\alpha_{n}-\alpha_{n} \beta_{n}(1-\alpha)\right)\right]\left\|x_{n}-p\right\| } \\
& +\left[\beta_{n}+\alpha_{n}-\alpha_{n} \beta_{n}(1-\alpha)\right]\|f(p)-p\| \\
\leq & \max \left\{\frac{1}{1-\alpha}\|f(p)-p\|,\left\|x_{n}-p\right\|\right\} .
\end{aligned}
$$

Using the induction method, we have

$$
\left\|x_{n}-p\right\| \leq \max \left\{\frac{1}{1-\alpha}\|f(p)-p\|,\left\|x_{0}-p\right\|\right\}, \quad n \geq 0,
$$

which implies that $\left\{x_{n}\right\},\left\{f\left(x_{n}\right)\right\},\left\{y_{n}\right\}$, and $\left\{f\left(y_{n}\right)\right\}$ are all bounded. Since $\left\|S_{r_{n}} x_{n}-p\right\| \leq\left\|x_{n}-p\right\|$, then $\left\{S_{r_{n}} x_{n}\right\}$ is bounded. Following the conditions of (i) and (ii), we obtain that

$$
\begin{aligned}
\left\|x_{n+1}-y_{n}\right\| & =\beta_{n}\left\|f\left(y_{n}\right)-y_{n}\right\| \longrightarrow 0, \quad \text { as } n \longrightarrow \infty \\
\left\|y_{n}-S_{r_{n}} x_{n}\right\| & =\alpha_{n}\left\|f\left(x_{n}\right)-S_{r_{n}} x_{n}\right\| \longrightarrow 0, \quad \text { as } n \longrightarrow \infty .
\end{aligned}
$$

Step 2. We show that $\left\|x_{n+1}-x_{n}\right\| \rightarrow 0$. that

For this, we estimate $y_{n+1}-y_{n}$ first. From (16), we know

$$
\begin{gathered}
y_{n}=\alpha_{n} f\left(x_{n}\right)+\left(1-\alpha_{n}\right) S_{r_{n}} x_{n}, \\
y_{n-1}=\alpha_{n-1} f\left(x_{n-1}\right)+\left(1-\alpha_{n-1}\right) S_{r_{n-1}} x_{n-1} .
\end{gathered}
$$

Then simple calculations show that

$$
\begin{aligned}
y_{n}-y_{n-1}= & \left(1-\alpha_{n}\right)\left(S_{r_{n}} x_{n}-S_{r_{n-1}} x_{n-1}\right) \\
& +\alpha_{n}\left(f\left(x_{n}\right)-f\left(x_{n-1}\right)\right) \\
& +\left(\alpha_{n}-\alpha_{n-1}\right)\left(f\left(x_{n-1}\right)-S_{r_{n-1}} x_{n-1}\right) .
\end{aligned}
$$

It follows from (25) that

$$
\begin{aligned}
& \left\|y_{n}-y_{n-1}\right\| \\
& \leq\left(1-\alpha_{n}\right)\left\|S_{r_{n}} x_{n}-S_{r_{n-1}} x_{n-1}\right\|+\alpha_{n} \alpha\left\|x_{n}-x_{n-1}\right\| \\
& \quad+\left|\alpha_{n}-\alpha_{n-1}\right|\left\|f\left(x_{n-1}\right)-S_{r_{n-1}} x_{n-1}\right\| .
\end{aligned}
$$

In view of Lemma 3, we have

$$
J_{r_{n}}^{A} x_{n}=J_{r_{n-1}}^{A}\left(\frac{r_{n-1}}{r_{n}} x_{n}+\left(1-\frac{r_{n-1}}{r_{n}}\right) J_{r_{n}}^{A} x_{n}\right) .
$$


If $r_{n-1} \leq r_{n}$, then

$$
\begin{aligned}
& \left\|J_{r_{n}}^{A} x_{n}-J_{r_{n-1}}^{A} x_{n-1}\right\| \\
& \quad=\left\|J_{r_{n-1}}^{A}\left(\frac{r_{n-1}}{r_{n}} x_{n}+\left(1-\frac{r_{n-1}}{r_{n}}\right) J_{r_{n}}^{A} x_{n}\right)-J_{r_{n-1}}^{A} x_{n-1}\right\| \\
& \quad \leq\left\|\frac{r_{n-1}}{r_{n}} x_{n}+\left(1-\frac{r_{n-1}}{r_{n}}\right) J_{r_{n}}^{A} x_{n}-x_{n-1}\right\| \\
& \quad \leq\left\|\frac{r_{n-1}}{r_{n}}\left(x_{n}-x_{n-1}\right)+\left(1-\frac{r_{n-1}}{r_{n}}\right)\left(J_{r_{n}}^{A} x_{n}-x_{n-1}\right)\right\| \\
& \quad \leq \frac{r_{n-1}}{r_{n}}\left\|x_{n}-x_{n-1}\right\|+\frac{r_{n}-r_{n-1}}{r_{n}}\left\|J_{r_{n}}^{A} x_{n}-x_{n-1}\right\| \\
& \quad \leq\left\|x_{n}-x_{n-1}\right\|+\frac{r_{n}-r_{n-1}}{\varepsilon}\left\|J_{r_{n}}^{A} x_{n}-x_{n-1}\right\| .
\end{aligned}
$$

Similarly,

$$
\begin{aligned}
\left\|J_{r_{n}}^{B} x_{n}-J_{r_{n-1}}^{B} x_{n-1}\right\| \leq & \left\|x_{n}-x_{n-1}\right\| \\
& +\frac{r_{n}-r_{n-1}}{\varepsilon}\left\|J_{r_{n}}^{B} x_{n}-x_{n-1}\right\| .
\end{aligned}
$$

Thus, let $M=\sup \left\{(1 / \varepsilon)\left\|J_{r_{n}}^{A} x_{n}-x_{n-1}\right\|,(1 / \varepsilon)\left\|J_{r_{n}}^{B} x_{n}-x_{n-1}\right\|\right\}$; we have

$$
\begin{aligned}
& \left\|S_{r_{n}} x_{n}-S_{r_{n-1}} x_{n-1}\right\| \\
& \quad \leq(1-\lambda)\left\|J_{r_{n}}^{A} x_{n}-J_{r_{n-1}}^{A} x_{n-1}\right\|+\lambda\left\|J_{r_{n}}^{B} x_{n}-J_{r_{n-1}}^{B} x_{n-1}\right\| \\
& \quad \leq\left\|x_{n}-x_{n-1}\right\|+\left(r_{n}-r_{n-1}\right) M .
\end{aligned}
$$

Substituting (30) into (26) we get

$$
\begin{aligned}
\left\|y_{n}-y_{n-1}\right\| \leq & \left(1-\alpha_{n}\right)\left(\left\|x_{n}-x_{n-1}\right\|+\left(r_{n}-r_{n-1}\right) M\right) \\
& +\alpha_{n} \alpha\left\|x_{n}-x_{n-1}\right\| \\
& +\left|\alpha_{n}-\alpha_{n-1}\right|\left\|f\left(x_{n-1}\right)-S_{r_{n-1}} x_{n-1}\right\| \\
\leq & \left(1-\alpha_{n}(1-\alpha)\right)\left\|x_{n}-x_{n-1}\right\| \\
& +\left(1-\alpha_{n}\right)\left(r_{n}-r_{n-1}\right) M \\
& +\left|\alpha_{n}-\alpha_{n-1}\right|\left\|f\left(x_{n-1}\right)-S_{r_{n-1}} x_{n-1}\right\| \\
\leq & \left(1-\alpha_{n}(1-\alpha)\right)\left\|x_{n}-x_{n-1}\right\| \\
& +M_{1}\left(\left|\alpha_{n}-\alpha_{n-1}\right|+\left|r_{n}-r_{n-1}\right|\right)
\end{aligned}
$$

where $M_{1}$ is a constant such that

$$
M_{1}>\max \left\{M,\left\|f\left(x_{n-1}\right)-S_{r_{n-1}} x_{n-1}\right\|\right\} .
$$

On the other hand, we have

$$
\begin{gathered}
x_{n+1}=\beta_{n} f\left(y_{n}\right)+\left(1-\beta_{n}\right) y_{n}, \\
x_{n}=\beta_{n-1} f\left(y_{n-1}\right)+\left(1-\beta_{n-1}\right) y_{n-1} .
\end{gathered}
$$

Simple calculations show that

$$
\begin{aligned}
x_{n+1}-x_{n}= & \left(1-\beta_{n}\right)\left(y_{n}-y_{n-1}\right)+\beta_{n}\left(f\left(y_{n}\right)-f\left(y_{n-1}\right)\right) \\
& +\left(\beta_{n}-\beta_{n-1}\right)\left(f\left(y_{n-1}\right)-y_{n-1}\right) .
\end{aligned}
$$

It follows that

$$
\begin{aligned}
\left\|x_{n+1}-x_{n}\right\| \leq & \left(1-\beta_{n}\right)\left\|y_{n}-y_{n-1}\right\|+\beta_{n} \alpha\left\|y_{n}-y_{n-1}\right\| \\
& +\left|\beta_{n}-\beta_{n-1}\right|\left\|f\left(y_{n-1}\right)-y_{n-1}\right\| \\
= & \left(1-\beta_{n}(1-\alpha)\right)\left\|y_{n}-y_{n-1}\right\| \\
& +\left|\beta_{n}-\beta_{n-1}\right|\left\|f\left(y_{n-1}\right)-y_{n-1}\right\| .
\end{aligned}
$$

Substituting (31) into (35) we get

$$
\begin{aligned}
& \left\|x_{n+1}-x_{n}\right\| \\
& \leq \quad\left(1-\beta_{n}(1-\alpha)\right)\left\{\left(1-\alpha_{n}(1-\alpha)\right)\left\|x_{n}-x_{n-1}\right\|\right. \\
& \left.\quad+M_{1}\left(\left|\alpha_{n}-\alpha_{n-1}\right|+\left|r_{n}-r_{n-1}\right|\right)\right\} \\
& \quad+\left|\beta_{n}-\beta_{n-1}\right|\left\|f\left(y_{n-1}\right)-y_{n-1}\right\| \\
& \leq \quad\left(1-\alpha_{n}(1-\alpha)\right)\left\|x_{n}-x_{n-1}\right\| \\
& \quad+M_{2}\left(\left|\alpha_{n}-\alpha_{n-1}\right|+\left|\beta_{n}-\beta_{n-1}\right|+\left|r_{n}-r_{n-1}\right|\right),
\end{aligned}
$$

where $M_{2}$ is a constant such that

$$
M_{2}>\max \left\{\left\|f\left(y_{n-1}\right)-y_{n-1}\right\|, M_{1}\right\} .
$$

From conditions (i)-(iii), we have that

$$
\begin{gathered}
\sum_{n=1}^{\infty} \alpha_{n}=\infty, \quad \alpha_{n} \longrightarrow 0, \text { as } n \longrightarrow \infty, \\
\sum_{n=1}^{\infty}\left(\left|\alpha_{n}-\alpha_{n-1}\right|+\left|\beta_{n}-\beta_{n-1}\right|+\left|r_{n}-r_{n-1}\right|\right)<+\infty .
\end{gathered}
$$

Hence, noticing (36) and applying Lemma 2, we obtain $\left\|x_{n+1}-x_{n}\right\| \rightarrow 0$. Then by (22) we obtain

$$
\left\|x_{n}-y_{n}\right\| \leq\left\|x_{n+1}-x_{n}\right\|+\left\|x_{n+1}-y_{n}\right\| \longrightarrow 0, \quad(n \longrightarrow \infty) .
$$

Step 3. We prove that $\left\|x_{n}-S_{r} x_{n}\right\| \rightarrow 0,\left\|y_{n}-S_{r} y_{n}\right\| \rightarrow 0$. In fact, since

$$
\left\|x_{n}-S_{r_{n}} x_{n}\right\| \leq\left\|x_{n}-x_{n+1}\right\|+\left\|x_{n+1}-y_{n}\right\|+\left\|y_{n}-S_{r_{n}} x_{n}\right\|,
$$


Abstract and Applied Analysis

5

from (22) and (23), we have $\left\|x_{n}-S_{r_{n}} x_{n}\right\| \rightarrow 0$. Then

$$
\begin{aligned}
\left\|S_{r_{n}} x_{n}-S_{r} x_{n}\right\| & \\
\leq & (1-\lambda)\left\|J_{r_{n}}^{A} x_{n}-J_{r}^{A} x_{n}\right\|+\lambda\left\|J_{r_{n}}^{B} x_{n}-J_{r}^{B} x_{n}\right\| \\
\leq & (1-\lambda)\left\|J_{r}^{A}\left(\frac{r}{r_{n}} x_{n}+\left(1-\frac{r}{r_{n}}\right) J_{r_{n}}^{A} x_{n}\right)-J_{r}^{A} x_{n}\right\| \\
& +\lambda\left\|J_{r}^{B}\left(\frac{r}{r_{n}} x_{n}+\left(1-\frac{r}{r_{n}}\right) J_{r_{n}}^{B} x_{n}\right)-J_{r}^{B} x_{n}\right\| \\
\leq & (1-\lambda)\left\|\left(\frac{r}{r_{n}} x_{n}+\left(1-\frac{r}{r_{n}}\right) J_{r_{n}}^{A} x_{n}\right)-x_{n}\right\| \\
& +\lambda\left\|\left(\frac{r}{r_{n}} x_{n}+\left(1-\frac{r}{r_{n}}\right) J_{r_{n}}^{B} x_{n}\right)-x_{n}\right\| \\
\leq & \left|1-\frac{r}{r_{n}}\right| \max \left\{\left\|J_{r_{n}}^{A} x_{n}-x_{n}\right\|,\left\|J_{r_{n}}^{B} x_{n}-x_{n}\right\|\right\} \longrightarrow 0 .
\end{aligned}
$$

Hence, we have

$$
\begin{aligned}
& \left\|x_{n}-S_{r} x_{n}\right\| \\
& \quad \leq\left\|x_{n}-S_{r_{n}} x_{n}\right\|+\left\|S_{r_{n}} x_{n}-S_{r} x_{n}\right\| \longrightarrow 0, \quad n \longrightarrow \infty, \\
& \left\|y_{n}-S_{r} y_{n}\right\| \\
& \quad \leq\left\|x_{n}-y_{n}\right\|+\left\|x_{n}-S_{r} x_{n}\right\|+\left\|S_{r} y_{n}-S_{r} x_{n}\right\| \\
& \quad \leq 2\left\|x_{n}-y_{n}\right\|+\left\|x_{n}-S_{r} x_{n}\right\| \longrightarrow 0, \quad n \rightarrow \infty .
\end{aligned}
$$

Step 4. We show that $\lim \sup _{n \rightarrow \infty}\left\langle(I-f) p, J\left(y_{n}-p\right)\right\rangle \leq 0$, $\limsup _{n \rightarrow \infty}\left\langle(I-f) p, J\left(x_{n+1}-p\right)\right\rangle \leq 0$.

To prove this, let $\left\{y_{n_{j}}\right\}$ be a subsequence of $\left\{y_{n}\right\}$ such that

$$
\begin{aligned}
& \limsup _{n \rightarrow \infty}\left\langle(I-f) p, J\left(y_{n}-p\right)\right\rangle \\
& \quad=\lim _{j \rightarrow \infty}\left\langle(I-f) p, J\left(y_{n_{j}}-p\right)\right\rangle .
\end{aligned}
$$

By Lemma $4, \lim _{t \rightarrow 0^{+}} z_{t}=p \in F\left(S_{r}\right)$, where $z_{t}=t f\left(z_{t}\right)+$ $(1-t) S_{r}\left(z_{t}\right)$. Then

$$
z_{t_{n}}-y_{n_{j}}=t_{n}\left(f\left(z_{t_{n}}\right)-y_{n_{j}}\right)+\left(1-t_{n}\right)\left(S_{r}\left(z_{t_{n}}\right)-y_{n_{j}}\right) .
$$

For each integer $n \geq 0$, let $t_{n} \in(0,1)$ such that

$$
t_{n} \longrightarrow 0, \quad \frac{\left\|S_{r} y_{n_{j}}-y_{n_{j}}\right\|}{t_{n}} \longrightarrow 0, \quad n \longrightarrow \infty .
$$

Using Lemma 1, we get

$$
\begin{aligned}
\| z_{t_{n}} & -y_{n_{j}} \|^{2} \\
\leq & \left(1-t_{n}\right)^{2}\left\|S_{r}\left(z_{t_{n}}\right)-y_{n_{j}}\right\|^{2} \\
& +2 t_{n}\left\langle f\left(z_{t_{n}}\right)-y_{n_{j}}, J\left(z_{t_{n}}-y_{n_{j}}\right)\right\rangle \\
\leq & \left(1-2 t_{n}+t_{n}^{2}\right)\left(\left\|S_{r}\left(z_{t_{n}}\right)-S_{r} y_{n_{j}}\right\|+\left\|y_{n_{j}}-S_{r} y_{n_{j}}\right\|\right)^{2} \\
& +2 t_{n}\left\langle f\left(z_{t_{n}}\right)-z_{t_{n}}, J\left(z_{t_{n}}-y_{n_{j}}\right)\right\rangle+2 t_{n}\left\|z_{t_{n}}-y_{n_{j}}\right\|^{2} \\
\leq & \left(1+t_{n}^{2}\right)\left\|z_{t_{n}}-y_{n_{j}}\right\|^{2}+\left(1+t_{n}\right)^{2}\left\|S_{r} y_{n_{j}}-y_{n_{j}}\right\| \\
& \times\left(2\left\|z_{t_{n}}-y_{n_{j}}\right\|+\left\|S_{r} y_{n_{j}}-y_{n_{j}}\right\|\right) \\
& +2 t_{n}\left\langle f\left(z_{t_{n}}\right)-z_{t_{n}}, J\left(z_{t_{n}}-y_{n_{j}}\right)\right\rangle
\end{aligned}
$$

and hence

$$
\begin{aligned}
& \left\langle z_{t_{n}}-f\left(z_{t_{n}}\right), J\left(z_{t_{n}}-y_{n_{j}}\right)\right\rangle \\
& \leq \frac{t_{n}}{2}\left\|z_{t_{n}}-y_{n_{j}}\right\|^{2}+\frac{\left(1+t_{n}\right)^{2}\left\|S_{r} y_{n_{j}}-y_{n_{j}}\right\|}{2 t_{n}} \\
& \quad \times\left(2\left\|z_{t_{n}}-y_{n_{j}}\right\|+\left\|S_{r} y_{n_{j}}-y_{n_{j}}\right\|\right) .
\end{aligned}
$$

Since $\left\{x_{n}\right\},\left\{z_{t_{n}}\right\}$, and $\left\{S_{r} y_{n}\right\}$ are bounded, then $\| S_{r} y_{n_{j}}-$ $y_{n_{j}} \| / 2 t_{n} \rightarrow 0$. Therefore,

$$
\limsup _{j \rightarrow \infty}\left\langle z_{t_{n}}-f\left(z_{t_{n}}\right), J\left(z_{t_{n}}-y_{n_{j}}\right)\right\rangle \leq 0 .
$$

We also know that

$$
\begin{aligned}
\langle p- & \left.f\left(z_{t_{n}}\right), J\left(y_{n_{j}}-p\right)\right\rangle \\
= & \left\langle p-f\left(z_{t_{n}}\right), J\left(y_{n_{j}}-p\right)-J\left(z_{t_{n}}-y_{n_{j}}\right)\right\rangle \\
& +\left\langle p-z_{t_{n}}, J\left(z_{t_{n}}-y_{n_{j}}\right)\right\rangle \\
& +\left\langle z_{t_{n}}-f\left(z_{t_{n}}\right), J\left(z_{t_{n}}-y_{n_{j}}\right)\right\rangle .
\end{aligned}
$$

Notice that $z_{t_{n}} \rightarrow p, p \in F\left(S_{r}\right), n \rightarrow \infty$, and $J$ is norm to weak* uniformly continuous on bounded subset of $E$; then we obtain

$$
\begin{array}{r}
\left\langle p-z_{t_{n}}, J\left(z_{t_{n}}-y_{n_{j}}\right)\right\rangle \longrightarrow 0, \quad n \longrightarrow \infty, \\
\left\langle p-f\left(z_{t_{n}}\right), J\left(y_{n_{j}}-p\right)-J\left(z_{t_{n}}-y_{n_{j}}\right)\right\rangle \\
n \rightarrow 0, \\
n \rightarrow \infty .
\end{array}
$$


From (48), (49), and the two results mentioned above, we have

$$
\limsup _{n \rightarrow \infty}\left\langle(I-f) p, J\left(y_{n}-p\right)\right\rangle \leq 0
$$

Using (22) and the property of $J$, we obtain the result that

$$
\begin{aligned}
& \limsup _{n \rightarrow \infty}\left\langle(I-f) p, J\left(y_{n}-p\right)\right\rangle \\
& \quad=\limsup _{n \rightarrow \infty}\left\langle(I-f) p, J\left(x_{n+1}-p\right)\right\rangle \leq 0 .
\end{aligned}
$$

Step 5. $\lim _{n \rightarrow \infty}\left\|x_{n}-p\right\|=0$.

Using (16), we have

$$
\begin{aligned}
\left\|y_{n}-p\right\|^{2} \\
=\left\|\alpha_{n}\left(f\left(x_{n}\right)-p\right)+\left(1-\alpha_{n}\right)\left(S_{r} x_{n}-p\right)\right\|^{2} \\
=\|\left(1-\alpha_{n}\right)\left(S_{r} x_{n}-p\right)+\alpha_{n}\left(f\left(x_{n}\right)-f(p)\right) \\
\quad+\alpha_{n}(f(p)-p) \|^{2} \\
\leq\left(1-\alpha_{n}(1-\alpha)\right)\left\|x_{n}-p\right\|^{2} \\
\quad+2 \alpha_{n}\left\langle f(p)-p, J\left(y_{n}-p\right)\right\rangle .
\end{aligned}
$$

Applying Lemma 1, we obtain

$$
\begin{aligned}
&\left\|x_{n+1}-p\right\|^{2} \\
&=\left\|\beta_{n}\left(f\left(y_{n}\right)-p\right)+\left(1-\beta_{n}\right)\left(y_{n}-p\right)\right\|^{2} \\
&=\|\left(1-\beta_{n}\right)\left(y_{n}-p\right) \\
& \quad+\beta_{n}\left(f\left(y_{n}\right)-f(p)\right)+\beta_{n}(f(p)-p) \|^{2} \\
& \leq\left(1-\beta_{n}(1-\alpha)\right)\left\|y_{n}-p\right\|^{2} \\
&+2 \beta_{n}\left\langle f(p)-p, J\left(x_{n+1}-p\right)\right\rangle \\
& \leq\left(1-\alpha_{n}(1-\alpha)\right)\left(1-\beta_{n}(1-\alpha)\right)\left\|x_{n}-p\right\|^{2} \\
&+2 \alpha_{n}\left(1-\beta_{n}(1-\alpha)\right)\left\langle f(p)-p, J\left(y_{n}-p\right)\right\rangle \\
&+2 \beta_{n}\left\langle f(p)-p, J\left(x_{n+1}-p\right)\right\rangle \\
& \leq {\left[1-\left(\alpha_{n}+\beta_{n}\right)(1-\alpha)\right]\left\|x_{n}-p\right\|^{2} } \\
&+\alpha_{n} \beta_{n}(1-\alpha)^{2}\left\|x_{n}-p\right\|^{2} \\
&-2 \alpha_{n} \beta_{n}(1-\alpha)\left\langle f(p)-p, J\left(y_{n}-p\right)\right\rangle \\
&+ 2\left(\alpha_{n}+\beta_{n}\right)\left\langle f(p)-p, J\left(x_{n+1}-p\right)\right\rangle \\
&+ 2 \alpha_{n}\left\langle f(p)-p, J\left(y_{n}-p\right)-J\left(x_{n+1}-p\right)\right\rangle
\end{aligned}
$$

$$
\begin{aligned}
\leq & {\left[1-\left(\alpha_{n}+\beta_{n}\right)(1-\alpha)\right]\left\|x_{n}-p\right\|^{2} } \\
& +\left(\alpha_{n}+\beta_{n}\right) \frac{\alpha_{n} \beta_{n}}{\alpha_{n}+\beta_{n}}(1-\alpha)^{2} L^{2} \\
& +2\left(\alpha_{n}+\beta_{n}\right) \frac{\alpha_{n} \beta_{n}}{\alpha_{n}+\beta_{n}}(1-\alpha) L^{2} \\
& +2\left(\alpha_{n}+\beta_{n}\right)\left\langle f(p)-p, J\left(x_{n+1}-p\right)\right\rangle \\
& +\left(\alpha_{n}+\beta_{n}\right) \frac{2 \alpha_{n}}{\alpha_{n}+\beta_{n}} \\
& \times\left\langle f(p)-p, J\left(y_{n}-p\right)-J\left(x_{n+1}-p\right)\right\rangle,
\end{aligned}
$$

where $L=\sup _{n \geq 0}\left\{\left\|x_{n}-p\right\|,\left\|y_{n}-p\right\|,\|f(p)-p\|\right\}$. Put

$$
\begin{gathered}
\gamma_{n}=\left(\alpha_{n}+\beta_{n}\right)(1-\alpha), \\
\sigma_{n}=\frac{\alpha_{n} \beta_{n}}{\alpha_{n}+\beta_{n}}(1-\alpha) L^{2}+\frac{2 \alpha_{n} \beta_{n}}{\alpha_{n}+\beta_{n}} L^{2} \\
+\frac{2}{1-\alpha}\left\langle f(p)-p, J\left(x_{n+1}-p\right)\right\rangle \\
+\frac{\alpha_{n}}{\alpha_{n}+\beta_{n}} \frac{2}{1-\alpha} \\
\times\left\langle f(p)-p, J\left(y_{n}-p\right)-J\left(x_{n+1}-p\right)\right\rangle .
\end{gathered}
$$

From the conditions (i) and (ii), the result of Step 4, and the facts that $\alpha_{n} \beta_{n} /\left(\alpha_{n}+\beta_{n}\right) \rightarrow 0$ and $\left\langle f(p)-p, J\left(y_{n}-p\right)-\right.$ $\left.J\left(x_{n+1}-p\right)\right\rangle \rightarrow 0$, we know that $\gamma_{n} \rightarrow 0, \sum_{n=1}^{\infty} \gamma_{n}=+\infty$ and $\lim \sup _{n \rightarrow \infty} \sigma_{n} \leq 0$. In view of Lemma 2, (54) reduces to

$$
\left\|x_{n+1}-p\right\| \leq\left(1-\gamma_{n}\right)\left\|x_{n}-p\right\|+\gamma_{n} \sigma_{n}
$$

then we know that

$$
\lim _{n \rightarrow \infty}\left\|x_{n}-p\right\|=0
$$

This completes the proof.

Remark 8. If we modify (16) as follows:

$$
\begin{gathered}
y_{n}=\alpha_{n} f\left(x_{n}\right)+\left(1-\alpha_{n}\right) S_{r_{n}} x_{n}, \\
x_{n+1}=\beta_{n} f\left(x_{n}\right)+\left(1-\beta_{n}\right) y_{n}, \\
y_{n}=\alpha_{n} u+\left(1-\alpha_{n}\right) S_{r_{n}} x_{n}, \\
x_{n+1}=\beta_{n} f\left(y_{n}\right)+\left(1-\beta_{n}\right) y_{n},
\end{gathered}
$$

or

$$
\begin{gathered}
y_{n}=\alpha_{n} u+\left(1-\alpha_{n}\right) S_{r_{n}} x_{n}, \\
x_{n+1}=\beta_{n} f\left(y_{n}\right)+\left(1-\beta_{n}\right) S_{r_{n}} y_{n} .
\end{gathered}
$$

Then, imitating the proof of Theorem 7, we can also get the result of Theorem 7 . Therefore, from the compare of iterative scheme, the conclusions of $[10,11]$ are special cases of Theorem 7. 
Example 9. Next we study the following optimization problem:

$$
\min _{x \in C} h(x), \quad \min _{x \in C} k(x),
$$

where $C$ is an interior nonempty closed convex subset of a Hilbert space and $h, k: C \rightarrow R$ are two proper convex and lower semicontinuous functionals. To solve optimization problem (61), we will list the following well known results.

Proposition 10 (see [18]). Let $\varphi: C \rightarrow R$ be a proper convex and lower semicontinuous functional. Then

(i) $\partial \varphi: C \rightarrow E^{*}$ (d denotes the subdifferential in the sense of convex analysis) is a maximal monotone mapping;

(ii) $\partial \varphi\left(x_{0}\right)=\min _{x \in C} \varphi(x)$ if and only if $0 \in \partial \varphi\left(x_{0}\right)$.

In Hilbert space $\partial \varphi$ is a $m$-accretive mapping. Thus $A=\partial h, B=\partial k$ are two $m$-accretive mappings. Solving optimization problem (61) is equivalent to finding a common zero of $A$ and $B$.

Let

$$
\begin{gathered}
J_{r}^{A}=(I+r A)^{-1}=(I+r \partial h)^{-1}, \\
J_{r}^{B}=(I+r B)^{-1}=(I+r \partial k)^{-1}, \\
S_{r}=(1-\lambda) J_{r}^{A}+\lambda J_{r}^{B}, \quad \alpha_{n}=\beta_{n}=\frac{1}{n}, \\
r_{n}=\frac{r n}{n+1}, \quad(r>\varepsilon) .
\end{gathered}
$$

Then the conditions (i), (ii), and (iii) of Theorem 7 are satisfied. For arbitrary $f \in \Sigma_{C}$ the sequence $\left\{x_{n}\right\}$ generated by (16) converges strongly to a common zero of $A$ and $B$, which is also the solution of the optimization problem (60).

Theorem 11. Let $A, B$ be two accretive maps in $E$ with $A^{-1} 0 \cap$ $B^{-1} 0 \neq \varnothing$ and satisfying the following range conditions: $\overline{D(A)} \subseteq$ $C \subset R(I+r A) \cap R(I+r B), \overline{D(B)} \subseteq C \subset R(I+r A) \cap R(I+r B)$ which are convex. Let $f,\left\{\alpha_{n}\right\},\left\{\beta_{n}\right\},\left\{r_{n}\right\}$, and $\left\{x_{n}\right\},\left\{y_{n}\right\}$ be the same as those in Theorem 7. Let $\left\{x_{n}\right\}$ be a sequence generated by (16). Then $\left\{x_{n}\right\}$ converges strongly to $p \in A^{-1} 0 \cap B^{-1} 0$, where $p$ is the unique solution of the following variational inequality:

$$
\langle(I-f) p, J(p-q)\rangle \leq 0, \quad f \in \Sigma_{C}, q \in A^{-1} 0 \cap B^{-1} 0 .
$$

Theorem 12. Let $A, B$ be two accretive maps in $E$ with $F=$ $A^{-1} 0 \cap B^{-1} 0 \neq \varnothing$ and satisfying the following range conditions: $\overline{D(A)} \subseteq C \subset R(I+r A) \cap R(I+r B), \overline{D(B)} \subseteq C \subset R(I+r A) \cap R(I+$ $r B)$ which are convex. Let $f,\left\{\alpha_{n}\right\},\left\{\beta_{n}\right\}$, and $\left\{r_{n}\right\}$ be the same as those in Theorem 7. Let $g: C \rightarrow C$ be a weakly contractive mapping with the function $\psi$. Let $\left\{x_{n}\right\}$ be a sequence generated by

$$
\begin{aligned}
& y_{n}=\alpha_{n} g\left(x_{n}\right)+\left(1-\alpha_{n}\right) S_{r_{n}} x_{n}, \\
& x_{n+1}=\beta_{n} f\left(y_{n}\right)+\left(1-\beta_{n}\right) y_{n} .
\end{aligned}
$$

Then $\left\{x_{n}\right\}$ converges strongly to $p=Q(g(p)) \in A^{-1} 0 \cap B^{-1} 0$, where $Q$ is a sunny nonexpansive retraction from $C$ onto $F$.
Proof. Since $E$ is a uniformly smooth Banach space, then there is a sunny nonexpansive retraction $Q$ from $C$ onto $F$. Then $Q \circ g$ is a weakly contractive mapping of $C$ into itself. Indeed, for all $x, y \in C$,

$$
\begin{aligned}
\|Q(g(x))-Q(g(y))\| & \leq\|g(x)-g(y)\| \\
& \leq\|x-y\|-\psi(\|x-y\|) .
\end{aligned}
$$

Lemma 5 assures that there exists a unique element $p \in C$ such that $p=Q(g(p))$. Such $p \in C$ is an element of $A^{-1} 0 \cap$ $B^{-1} 0$. Now we define a iterative scheme as follows:

$$
\begin{aligned}
& z_{n}=\alpha_{n} g(p)+\left(1-\alpha_{n}\right) S_{r_{n}} w_{n}, \\
& w_{n+1}=\beta_{n} f\left(z_{n}\right)+\left(1-\beta_{n}\right) z_{n} .
\end{aligned}
$$

Let $w_{n}$ be the sequence generated by (66). Then Remark 8 (59) assures that $w_{n}$ converges strongly to $p=Q(g(p))$ as $n \rightarrow$ $\infty$. For any $n$, we have

$$
\begin{aligned}
\left\|x_{n+1}-w_{n+1}\right\| & \\
\leq & \beta_{n}\left\|f\left(y_{n}\right)-f\left(z_{n}\right)\right\|+\left(1-\beta_{n}\right)\left\|y_{n}-z_{n}\right\| \\
\leq & \left\|y_{n}-z_{n}\right\| \\
\leq & \alpha_{n}\left\|g\left(x_{n}\right)-g(p)\right\|+\left(1-\alpha_{n}\right)\left\|S_{r_{n}} x_{n}-S_{r_{n}} w_{n}\right\| \\
\leq & \alpha_{n}\left\|g\left(x_{n}\right)-g\left(w_{n}\right)\right\|+\alpha_{n}\left\|g\left(w_{n}\right)-g(p)\right\| \\
& +\left(1-\alpha_{n}\right)\left\|x_{n}-w_{n}\right\| \\
\leq & \left\|x_{n}-w_{n}\right\|-\alpha_{n} \psi\left(\left\|x_{n}-w_{n}\right\|\right) \\
& +\alpha_{n}\left(\left\|w_{n}-p\right\|-\psi\left(\left\|w_{n}-p\right\|\right)\right) \\
\leq & \left\|x_{n}-w_{n}\right\|-\alpha_{n} \psi\left(\left\|x_{n}-w_{n}\right\|\right)+\alpha_{n}\left\|w_{n}-p\right\| .
\end{aligned}
$$

Thus, we obtain for $s_{n}=\left\|x_{n}-w_{n}\right\|$ the following recursive inequality:

$$
s_{n+1}=s_{n}-\alpha_{n} \psi\left(s_{n}\right)+\alpha_{n}\left\|w_{n}-p\right\| .
$$

Since $\left\|w_{n}-p\right\| \rightarrow 0$, it follows from Lemma 6 that $\lim _{n \rightarrow \infty}\left\|x_{n}-w_{n}\right\|=0$. Hence

$$
\lim _{n \rightarrow \infty}\left\|x_{n}-p\right\| \leq \lim _{n \rightarrow \infty}\left(\left\|x_{n}-w_{n}\right\|+\left\|w_{n}-p\right\|\right)=0 .
$$

This completes the proof.

In virtue of the weakly contractive mapping $g$ being a contraction, using Theorem 12 we may obtain the following.

Corollary 13. Let $A, B$ be two accretive maps in $E$ with $F=$ $A^{-1} 0 \cap B^{-1} 0 \neq \varnothing$ and satisfying the following range conditions: $\overline{D(A)} \subseteq C \subset R(I+r A) \cap R(I+r B), \overline{D(B)} \subseteq C \subset R(I+r A) \cap R(I+$ $r B)$ which are convex. Let $f, g \in \Sigma_{C},\left\{\alpha_{n}\right\},\left\{\beta_{n}\right\}$, and $\left\{r_{n}\right\}$ be the same as those in Theorem 7. Let $\left\{x_{n}\right\}$ be a sequence generated by

$$
\begin{aligned}
& y_{n}=\alpha_{n} g\left(x_{n}\right)+\left(1-\alpha_{n}\right) S_{r_{n}} x_{n}, \\
& x_{n+1}=\beta_{n} f\left(y_{n}\right)+\left(1-\beta_{n}\right) y_{n} .
\end{aligned}
$$


Then $\left\{x_{n}\right\}$ converges strongly to $p=Q(g(p)) \in A^{-1} 0 \cap B^{-1} 0$, where $Q$ is a sunny nonexpansive retraction from $C$ onto $F$.

Example 14. Next we give an essential example.

Let $\Omega$ be an bounded domain in a Euclidean space $R^{N}$ with Lipschitz boundary $\Gamma$. Let $\phi: \Gamma \times R \rightarrow R$ be a given function shch that for each $x \in \Gamma$

(i) $\phi_{x}=\phi(x, \cdot): R \rightarrow R$ is a proper, convex, lower semicontinuous function with $\phi_{x}(0)=0$.

(ii) $\beta_{x}=\partial \phi_{x}$ (subdifferential of $\phi_{x}$ ) is the maximal monotone mapping on $R$ with $0 \in \beta_{x}(0)$ and for each $t \in R$, the function $x \in \Gamma \rightarrow\left(I+\lambda \beta_{x}\right)^{-1}(t) \in R$ is measurable for $\lambda>0$.

Let $\alpha: R^{N} \rightarrow R^{N}$ be a continuous, monotone function such that there exist constants $k_{1}, k_{2}$ satisfying (i) $|\alpha(\xi)| \leq$ $k_{1}|\xi|$ and (ii) $\langle\alpha(\xi), \xi\rangle \geq k_{2}|\xi|^{2}$ for each $\xi \in R^{N}$.

Definition 15 (see [19]). One first defines a mapping $A^{\alpha}$ : $H^{1}(\Omega) \rightarrow\left(H^{1}(\Omega)\right)^{*}\left(H^{1}(\Omega)\right.$ is a sobolev space) by

$$
\left(A^{\alpha} u, v\right)=\int_{\Omega}\langle\alpha(\operatorname{grad} u), \operatorname{grad} v\rangle d x
$$

for $u, v \in H^{1}(\Omega)$. Clearly $A^{\alpha}$ is an everywhere defined, monotone, demicontinuous operator from $H^{1}(\Omega)$ into $\left(H^{1}(\Omega)\right)^{*}$. Second one defines an operator $A_{p}^{\alpha}: L^{p}(\Omega) \rightarrow 2^{L^{p}(\Omega)}$ for $1<p<+\infty$ as follows.

(i) For $p \geq 2$ one defines the domain of $A_{p}^{\alpha}$ by

$$
\begin{gathered}
D\left(A_{p}^{\alpha}\right)=\left\{u \in L^{p}(\Omega): \text { there exists an } f \in L^{p}(\Omega)\right. \\
\text { such that } \left.A^{\alpha} u+\partial \Phi(u) \ni f\right\} .
\end{gathered}
$$

Here $\Phi(u)=\int_{\Gamma} \phi_{x}\left(\left.u\right|_{\Gamma}(x)\right) d \Gamma(x)$ is the proper, convex, l.s.c. function (see $\left[19\right.$, Lemma 3.1]). For $u \in D\left(A_{p}^{\alpha}\right)$ we set

$$
A_{p}^{\alpha}(u)=\left\{f \in L^{p}(\Omega) \mid A^{\alpha} u+\partial \Phi(u) \ni f\right\} .
$$

(ii) For $1<p<2$, one defines $A_{p}^{\alpha}$ as the $L^{p}$-closure of $A_{2}^{\alpha}$ defined in (i) above.

For the operator $A_{p}^{\alpha}$ one has following results.

Lemma 16 (see [19, Lemma 3.4]). $A_{p}^{\alpha}: L^{p}(\Omega) \rightarrow 2^{L^{p}(\Omega)}$ is $m$-accretive operator $(1<p<+\infty)$.

Lemma 17 (see [19, Proposition 3.2]). Let $f \in L^{p}(\Omega), u \in$ $L^{p}(\Omega)$ such that $f \in A_{p}^{\alpha} u$. Then

(i) $\operatorname{div}(\alpha(\operatorname{gradu}))=f$, a.e. on $\Omega$ and

(ii) $\langle n, \alpha(\operatorname{grad} u)\rangle \in \beta_{x}(u(x))$ for a.e. $x \in \Gamma$.

Lemma 18 (see [19, Proposition 3.3]). Let $\beta_{x} \equiv 0$ for $x \in \Gamma$. Then $\left(A_{p}^{\alpha}\right)^{-1} 0=\left\{u \in L^{p}(\Omega) \mid u=a\right.$ constant function $\}$.

Clearly for different $\alpha_{1}, \alpha_{2}, A_{p}^{\alpha_{1}}, A_{p}^{\alpha_{2}}$ are two $m$-accretive operators. The above results show that

$$
\begin{aligned}
\varnothing & \neq\left(A_{p}^{\alpha_{1}}\right)^{-1} 0 \cap\left(A_{p}^{\alpha_{2}}\right)^{-1} 0 \\
& =\left\{u \in L^{p}(\Omega) \mid u=\text { a constant function }\right\} .
\end{aligned}
$$

For the sake of finding a common zero, Theorems 7, 11, and 12 provided three different iterative algorithms. Therefore the study of a common zero of two accretive operators makes sense.

Remark 19. The results presented in this paper substantially improve and extend the results of Ceng et al. [10] from the following aspects.

(1) Theorems 7 and 12 extend the result on the iterative construction of the zero for a single accretive operator to the case of that for common zeros of two accretive operators. If we modify two accretive operators as finite accretive operators, then, imitating the proof of Theorem 7, we can also get the result of Theorem 7 .

(2) Our results include one or two different viscosity items. Remark 8 shows that the conclusions of Ceng et al. are special cases of this paper.

(3) The viscosity item is changed from a contractive mapping $f$ to weakly contractive mapping $g$ in Theorem 12 .

\section{Acknowledgments}

This work was supported by the National Natural Science Foundation of China (Grants no. 11101115, 11271106) and the Natural Science Foundation of Hebei Province (A2011201053).

\section{References}

[1] J. Diestel, Geometry of Banach Spaces, vol. 485 of Lecture Notes in Mathematics, Springer, Berlin, Germany, 1975.

[2] K. Goebel and S. Reich, Uniform Convexity, Hyperbolic Geometry, and Nonexpansive Mappings, vol. 83 of Monographs and Textbooks in Pure and Applied Mathematics, Marcel Dekker, New York, NY, USA, 1984.

[3] H. K. Xu, "Viscosity approximation methods for nonexpansive mappings," Journal of Mathematical Analysis and Applications, vol. 298, no. 1, pp. 279-291, 2004.

[4] R. E. Bruck and G. B. Passty, "Almost convergence of the infinite product of resolvents in Banach spaces," Nonlinear Analysis, vol. 3, no. 2, pp. 279-282, 1979.

[5] R. E. Bruck and S. Reich, "Nonexpansive projections and resolvents of accretive operators in Banach spaces," Houston Journal of Mathematics, vol. 3, no. 4, pp. 459-470, 1977.

[6] J. S. Jung and W. Takahashi, "Dual convergence theorems for the infinite products of resolvents in Banach spaces," Kodai Mathematical Journal, vol. 14, no. 3, pp. 358-365, 1991.

[7] J. S. Jung and W. Takahashi, "On the asymptotic behavior of infinite products of resolvents in Banach spaces," Nonlinear Analysis: Theory, Methods \& Applications, vol. 20, no. 5, pp. 469479, 1993.

[8] S. Reich, "On infinite products of resolvents," Atti della Accademia Nazionale dei Lincei, vol. 63, no. 5, pp. 338-340, 1977.

[9] S. Reich, "Weak convergence theorems for nonexpansive mappings in Banach spaces," Journal of Mathematical Analysis and Applications, vol. 67, no. 2, pp. 274-276, 1979. 
[10] L. C. Ceng, A. R. Khan, Q. H. Ansari, and J. C. Yao, "Strong convergence of composite iterative schemes for zeros of maccretive operators in Banach spaces," Nonlinear Analysis: Theory, Methods \& Applications, vol. 70, no. 5, pp. 1830-1840, 2009.

[11] R. Chen, Y. Liu, and X. Shen, "Iterative approximation of a zero of accretive operator in Banach space," Nonlinear Analysis: Theory, Methods \& Applications, vol. 71, no. 12, pp. e346-e350, 2009.

[12] J. S. Jung, "Convergence of composite iterative methods for finding zeros of accretive operators," Nonlinear Analysis: Theory, Methods \& Applications, vol. 71, no. 5-6, pp. 1736-1746, 2009.

[13] L. Hu and L. Liu, "A new iterative algorithm for common solutions of a finite family of accretive operators," Nonlinear Analysis: Theory, Methods \& Applications, vol. 70, no. 6, pp. 2344-2351, 2009.

[14] H. Zegeye and N. Shahzad, "Strong convergence theorems for a common zero of a countably infinite family of $\alpha$-inverse strongly accretive mappings," Nonlinear Analysis: Theory, Methods \& Applications, vol. 71, no. 1-2, pp. 531-538, 2009.

[15] Y. I. Alber and S. Guerre-Delabriere, "Principle of weakly contractive maps in Hilbert spaces," in New Results in Operator Theory and Its Applications, vol. 98 of Operator Theory: Advances and Applications, pp. 7-22, 1997.

[16] Y. I. Alber, S. Guerre-Delabriere, and L. Zelenko, "The principle of weakly contractive mappings in metric spaces," Communications on Applied Nonlinear Analysis, vol. 5, no. 1, pp. 45-68, 1998.

[17] B. E. Rhodes, "Some theorems on weakly contractive maps," Nonlinear Analysis: Theory, Methods \& Applications, vol. 47, no. 4, pp. 2683-2693, 2001.

[18] D. Pascali and S. Sburlan, Nonlinear Mappings of Monotone Type, Sijthoff and Noordholff International Publishers, Alphen aan den Rijn, The Netherlands, 1978.

[19] B. D. Calvert and C. P. Gupta, "Nonlinear elliptic boundary value problems in $L^{p}$-spaces and sums of ranges of accretive operators," Nonlinear Analysis, vol. 2, no. 1, pp. 1-26, 1978. 


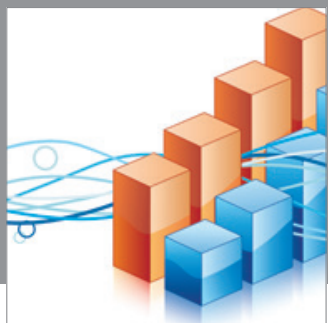

Advances in

Operations Research

mansans

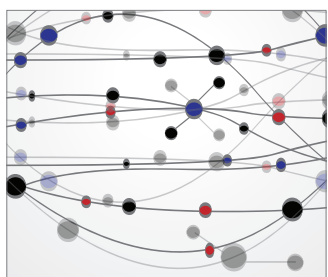

The Scientific World Journal
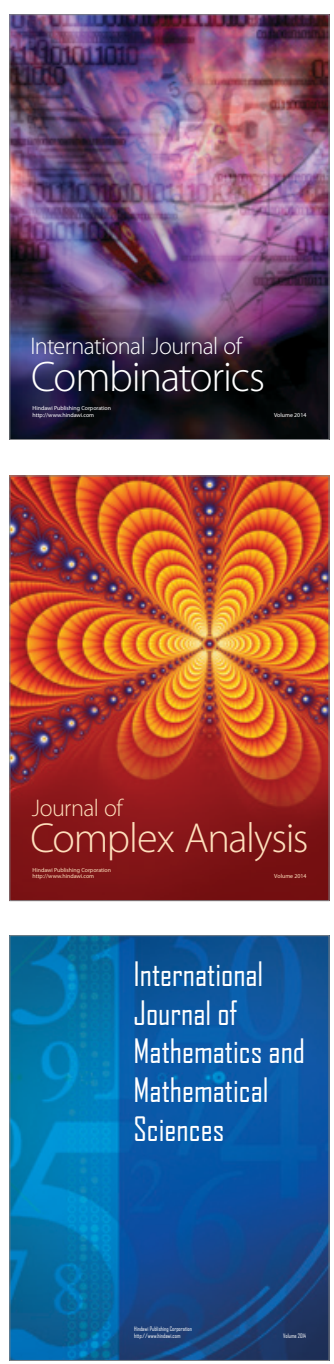
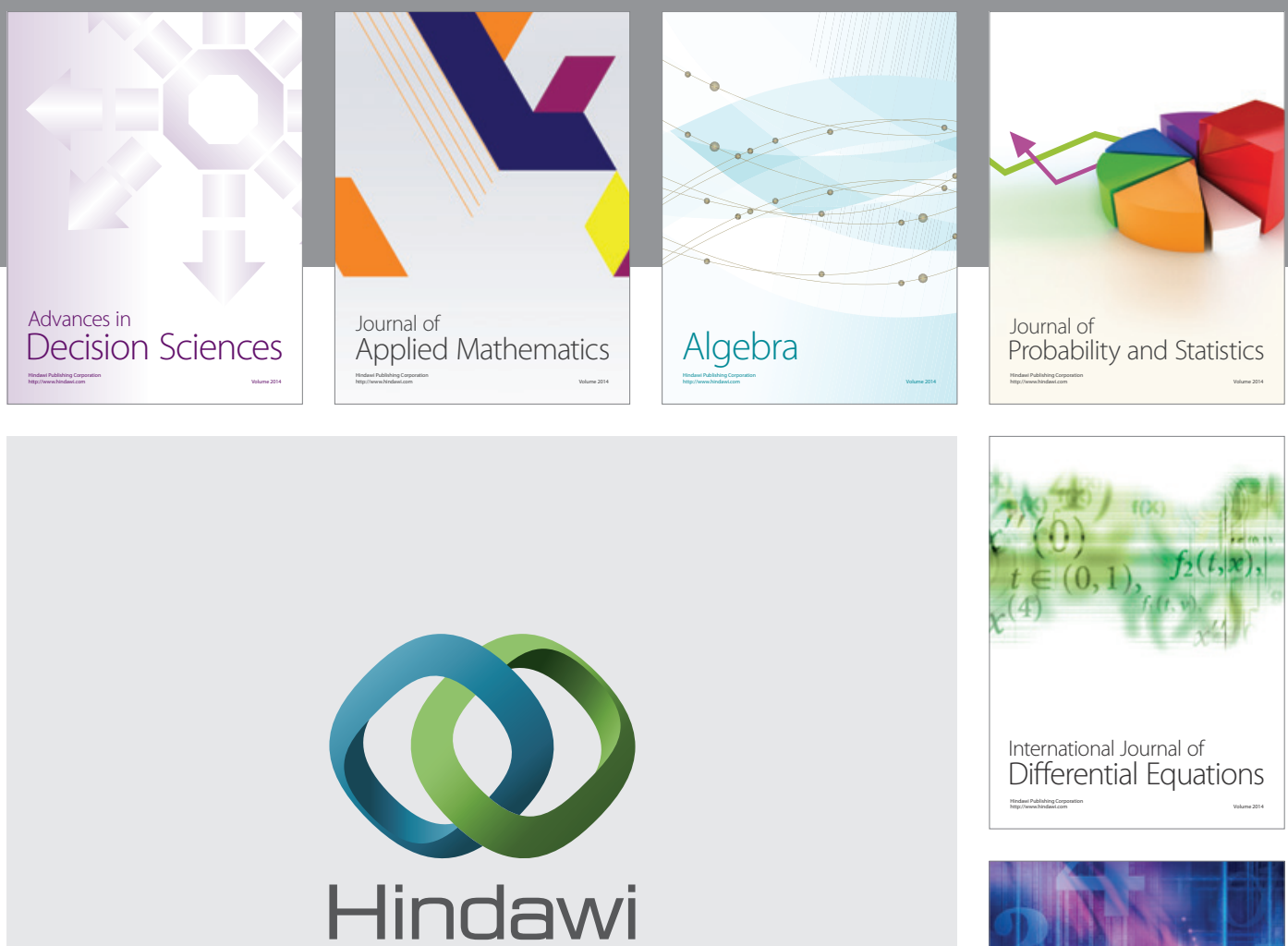

Submit your manuscripts at http://www.hindawi.com
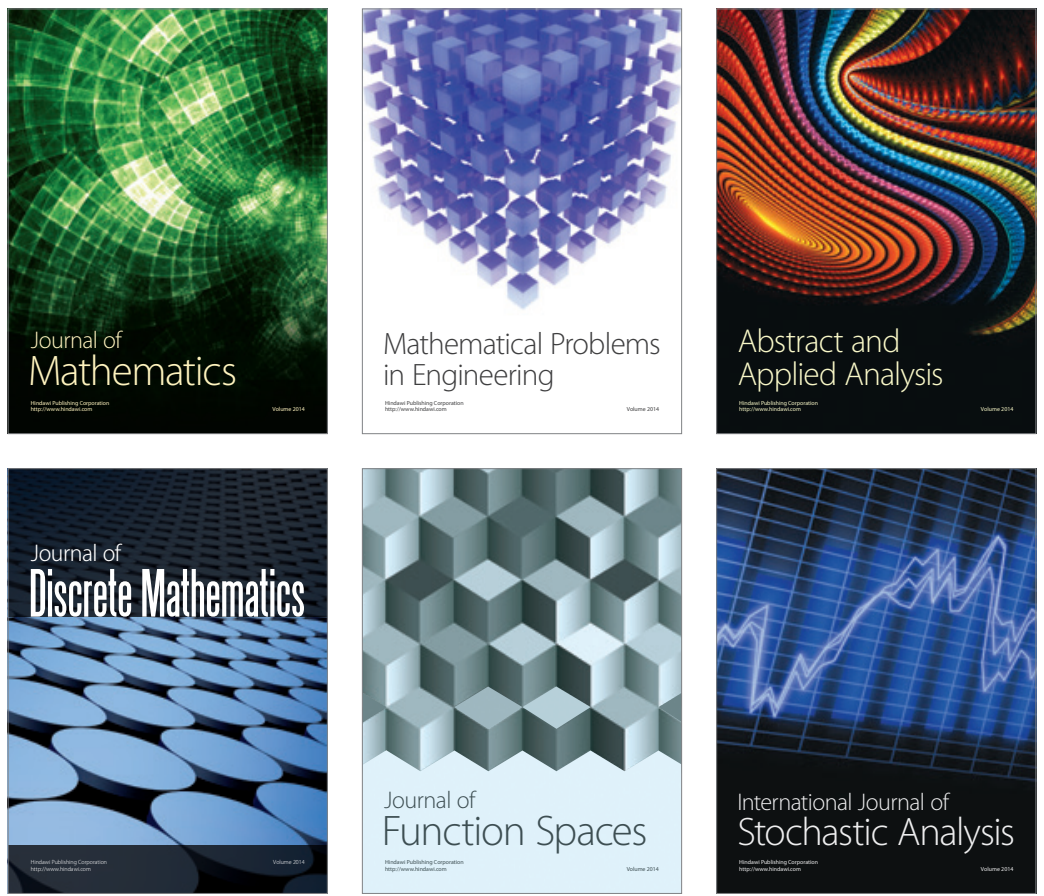

Journal of

Function Spaces

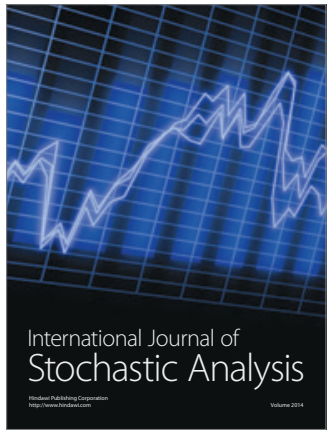

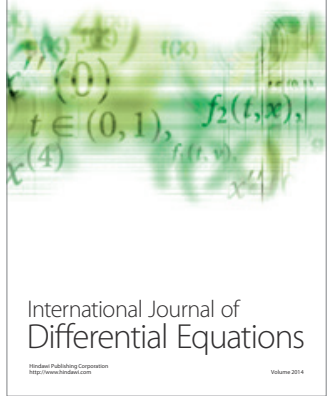
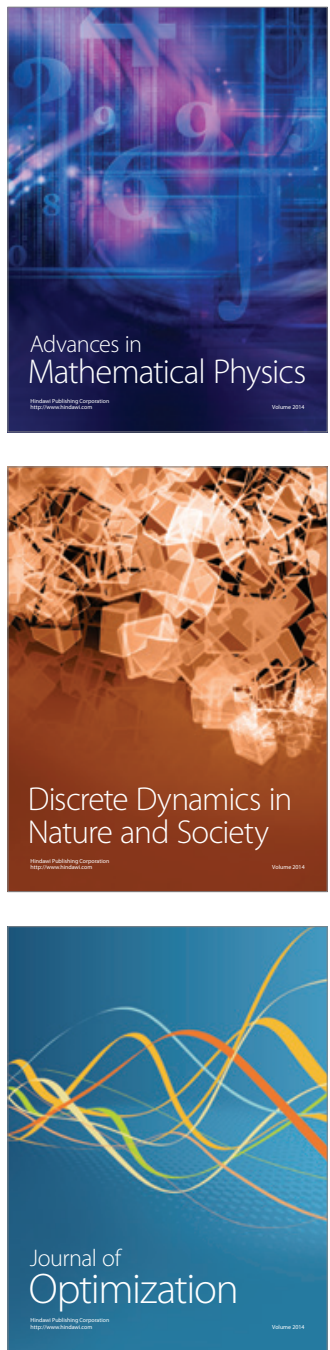